\title{
Image Mining of Geotagged Images Extracted from Cloud for Prediction of Next Probable Location
}

\author{
Vijay Bagdi, Sulabha Patil, Dr. R.V.Dharaskar \\ Student TPCET, Nagpur \\ Asst. Prof. Dept. MTech(CSE) TGPCET, Nagpur \\ Director MPGI, Nanded
}

\begin{abstract}
We propose system that helps to find the next probable location using attributes of geotagged images. Our approach goes in direction of developing an unattended system able to extract forensically viable information embedded with geotagged images using CBIR (Content Based Image Retrieval) and using that information it predicts next probable location. We use Haar and D4 wavelet to decompose color images into multilevel scale and wavelet coefficients, with which we perform image feature extraction and similarity match by means of F-norm theory.
\end{abstract}

Keywords: - Geotagged Images, CBIR, Image Retrieval, Prediction

\section{INTRODUCTION}

Content Based Image Retrieval is process of retrieving desired images from huge database based on features of images [1]. . Over the past four years, tourist databases have received particular attention and have been featured on the front page of newspapers. These databases have proven extremely useful in verifying or falsifying the involvement of a person or an object as a source of evidence material in a crime, and have led to the resolution of old cases [7]. Tourist databases are also playing an increasingly prominent role in the forensic literature. Research into forensic image databases is a rapidly expanding field of scientific endeavor that has a direct impact on the number of criminal cases solved. Throughout the 20th century, many databases were available in the form of paper files or photographs.

This project addresses the growing need for incorporating scientifically based approaches to conducting forensic analysis in the digital world rather than developing digital technologies and then adapting them to benefit from forensic analysis techniques.

This paper focused on digital forensic for finding out next probable location of an individual using CBIR as forensic tool.

II. PROPOSED METHODOLOGY

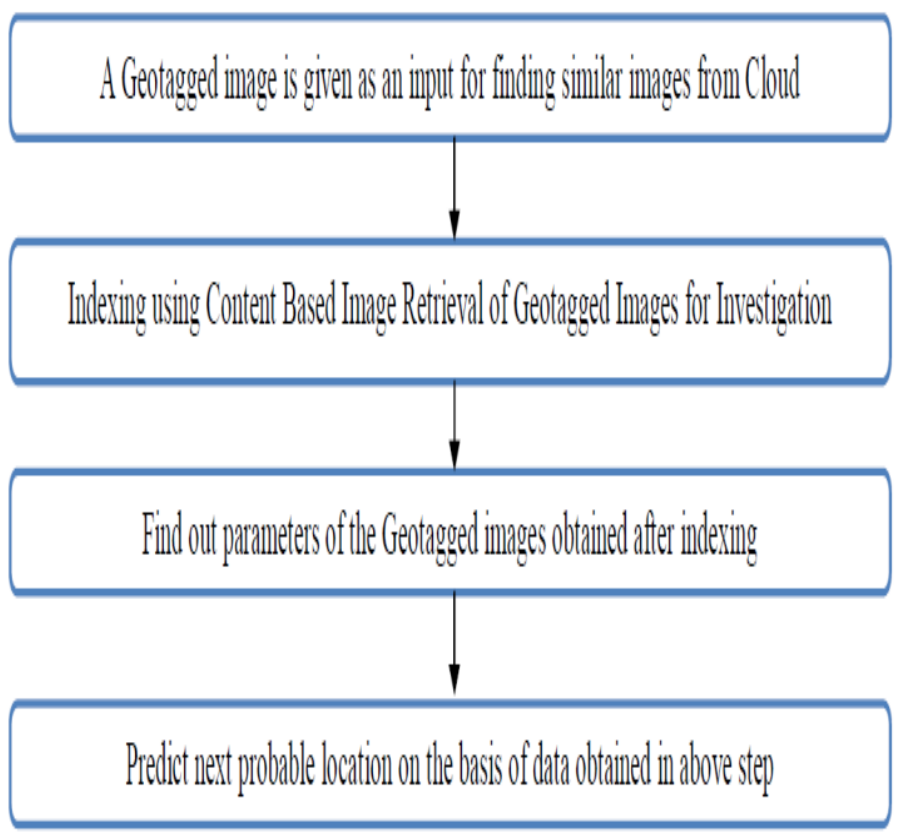


III.

PROPOSED ALGORITHM

1. Get GPS information from input image

2. Calculate cluster and dist of image from content.

3. From the above information get the matching history data using MYSQL query :Select *,3956 * $2 * \operatorname{ASIN}(\operatorname{SQRT}($ POWER(SIN(("+ historyData.getLat ()$+"$ - abs (lat)) * pi()/180 / 2),2)+" + "COS("+ historyData.getLat ()$+" *$ pi( $) / 180) * \operatorname{COS}($ abs $($ lat $) \quad * \quad$ pi( $) / 180) \quad * \quad P O W E R(\operatorname{SIN}(("+$ historyData.getLng()+" - lng) * pi()/180 / 2), 2)))" + " as distance From history_tbl having distance < 20 ORDER By distance

4. Find the matching cluster data, from cluster present in cloud (passed input as 'searched image')

5. First collect image result data from matching db data (step 3 input)

6. Now remove matching imgResultDb form searchForNextLocation result (removed the matching images 4 \& 5)

7. Collect history data from filter images (input pass 6)

8. Find next location (input 1 and 7)

\section{EXPERIMENTS AND RESULTS}

\subsection{Performance evaluation}

The performance of retrieval system is measured using the standard procedure in terms of the precision and recall values [5]. Recall measures the ability of the system to retrieve all the models that are relevant, while precision measures the ability of the system to retrieve only the models that are relevant. They are defined as

$$
\begin{aligned}
& \text { Precision }(P)=\frac{\text { Number of relevant images retrieved }}{\text { Total number of images retrieved }} \\
& \text { Recall }(R)=\frac{\text { Number of relevant images retrieved }}{\text { Total number of relevant images }}
\end{aligned}
$$

The number of relevant items retrieved is the number of the returned images that are similar to the query image in this case. The number of relevant items in the collection is the number of images that are in the same particular category with the query image. The total number of items retrieved is the number of images that are returned by the search engine [2].

\begin{tabular}{|c|c|c|}
\hline & $\begin{array}{c}\text { Precision Rate } \\
\text { (Top 5) }\end{array}$ & $\begin{array}{c}\text { Precision Rate } \\
\text { (Top 10) }\end{array}$ \\
\hline Vijay & 1 & 1 \\
\hline Sanket & 1 & 0.9 \\
\hline Jitesh & 1 & 1 \\
\hline Vishal & 1 & 0.9 \\
\hline Amit & 0.8 & 0.7 \\
\hline
\end{tabular}

Table 4.1.1: Precision Rate for Top 5 and Top 10 images

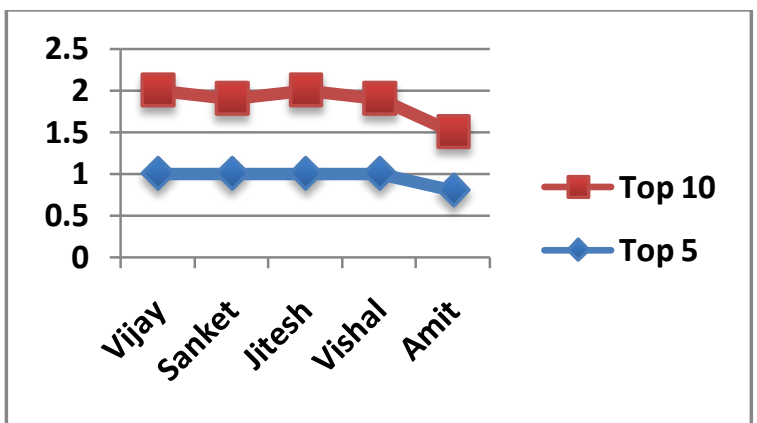

Figure 4.1.1: Precision Rate for Top 10 and Top 5 images

\begin{tabular}{|c|c|}
\hline & Retrieval Speed (s) \\
\hline Vijay & 2.9 \\
\hline Sanket & 3.8 \\
\hline Jitesh & 2.3 \\
\hline Vishal & 4.7 \\
\hline Amit & 4.2 \\
\hline
\end{tabular}

Table 4.1.2: Retrieval Speed 


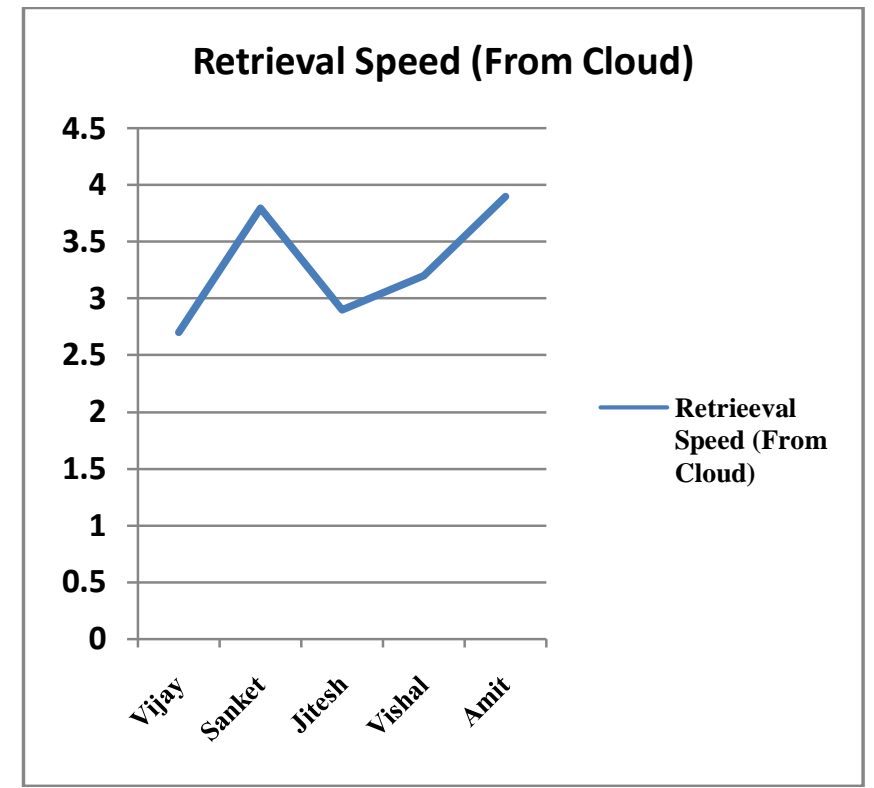

Figure 4.1.2: Graph Showing Retrieval Speed in Sec (From Cloud)

\subsection{Experimental Results}

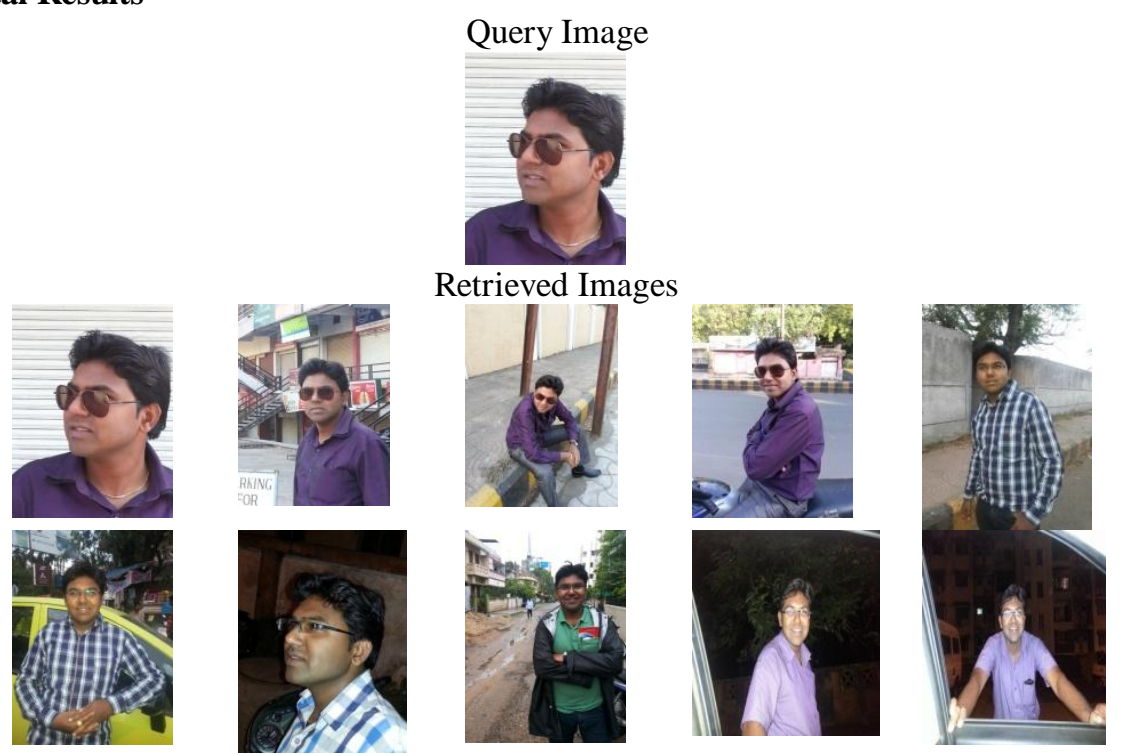

Figure 4.2.1: Vijay Query, 10 matches from top 10 retrieved images

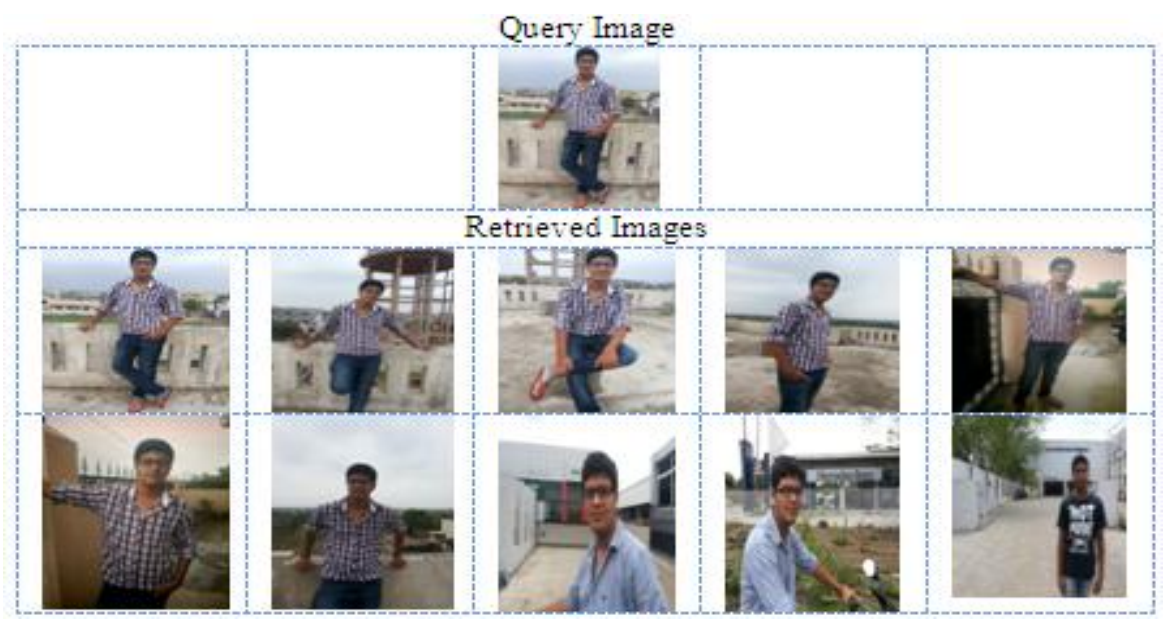

Figure 4.2.2: Sanket Query, 10 matches from top 10 retrieved images 


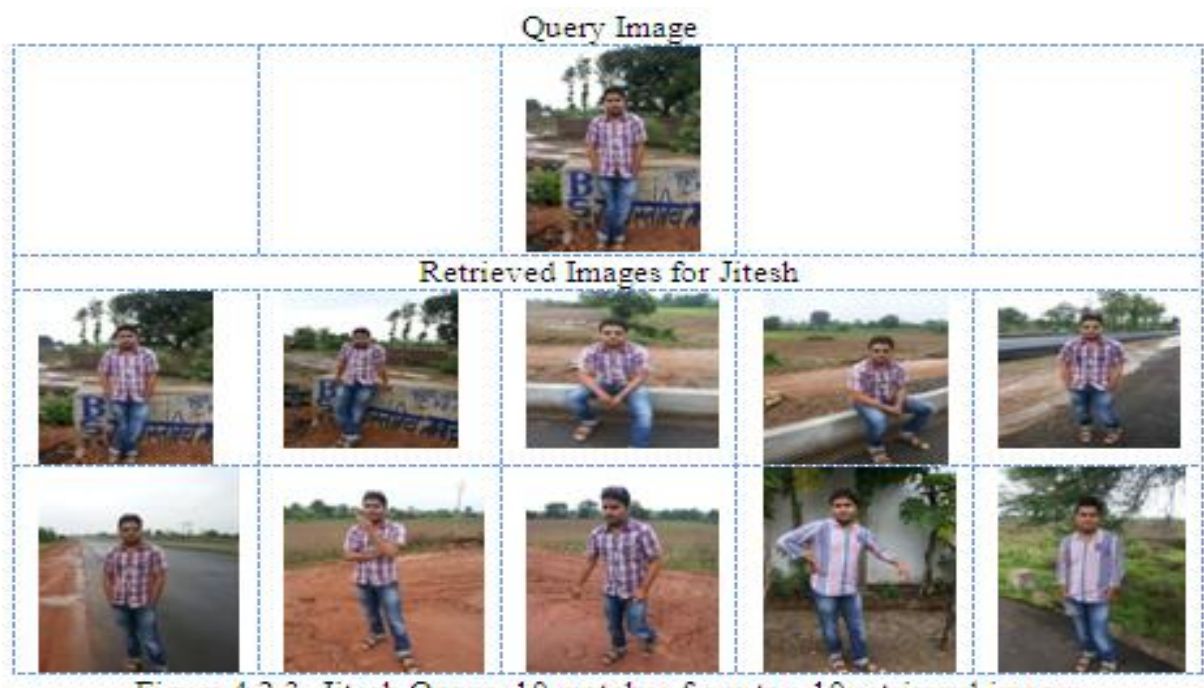

Figure 4.2.3: Jitesh Query, 10 matches from top 10 retrieved images

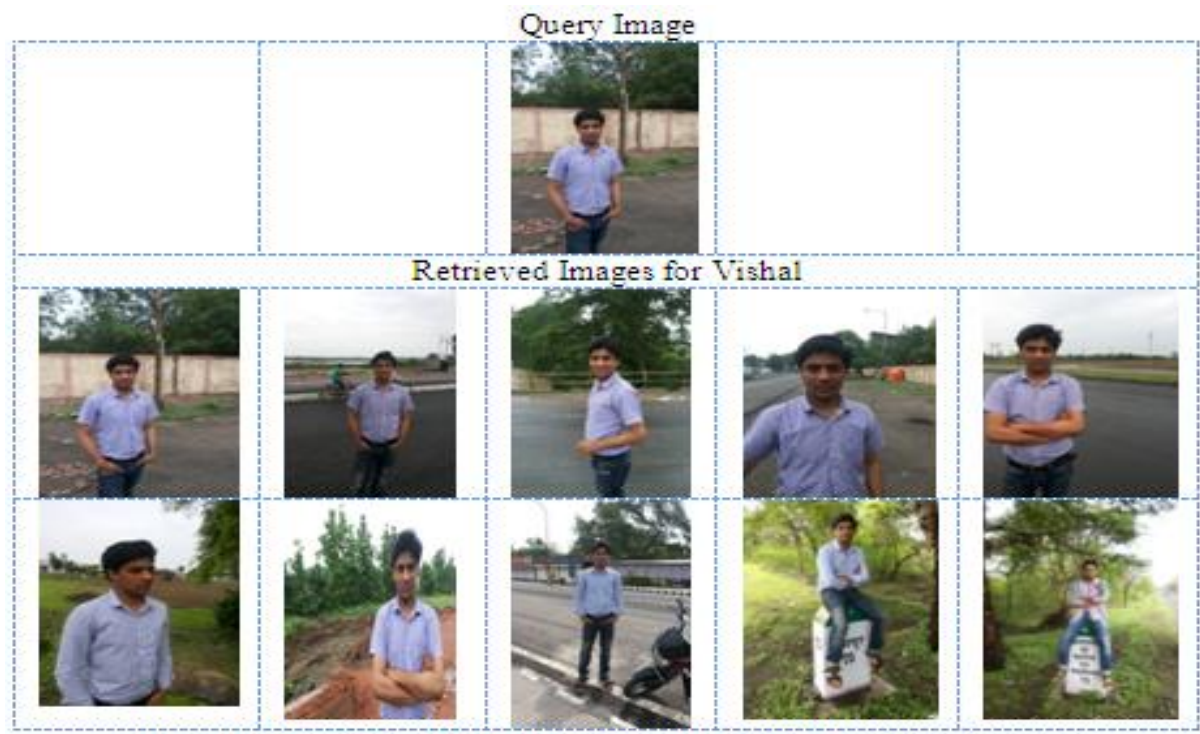

Figure 4.2.4: Vishal Query, 10 matches from top 10 retrieved images

Query Image

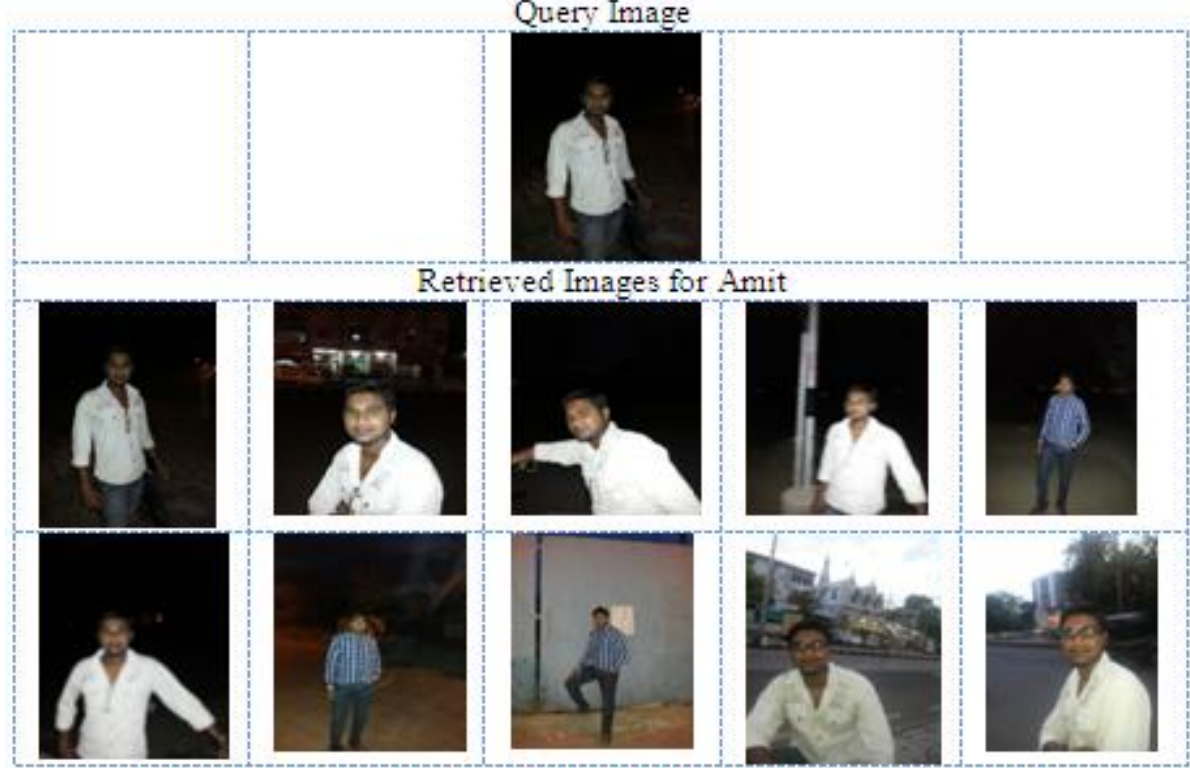

Figure 4.2.5: Amit Query, 10 matches from top 10 retrieved images 


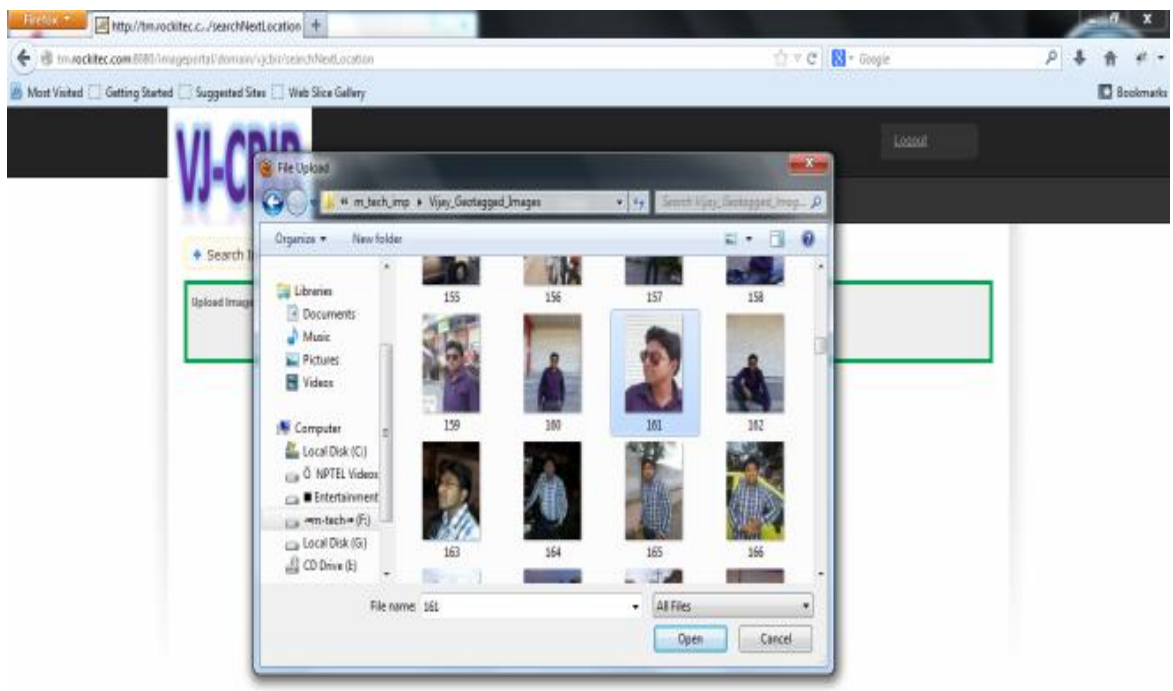

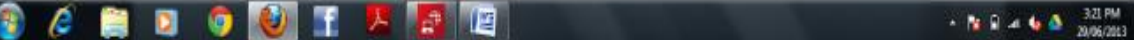

Figure 4.2.6: Selection of Input Image

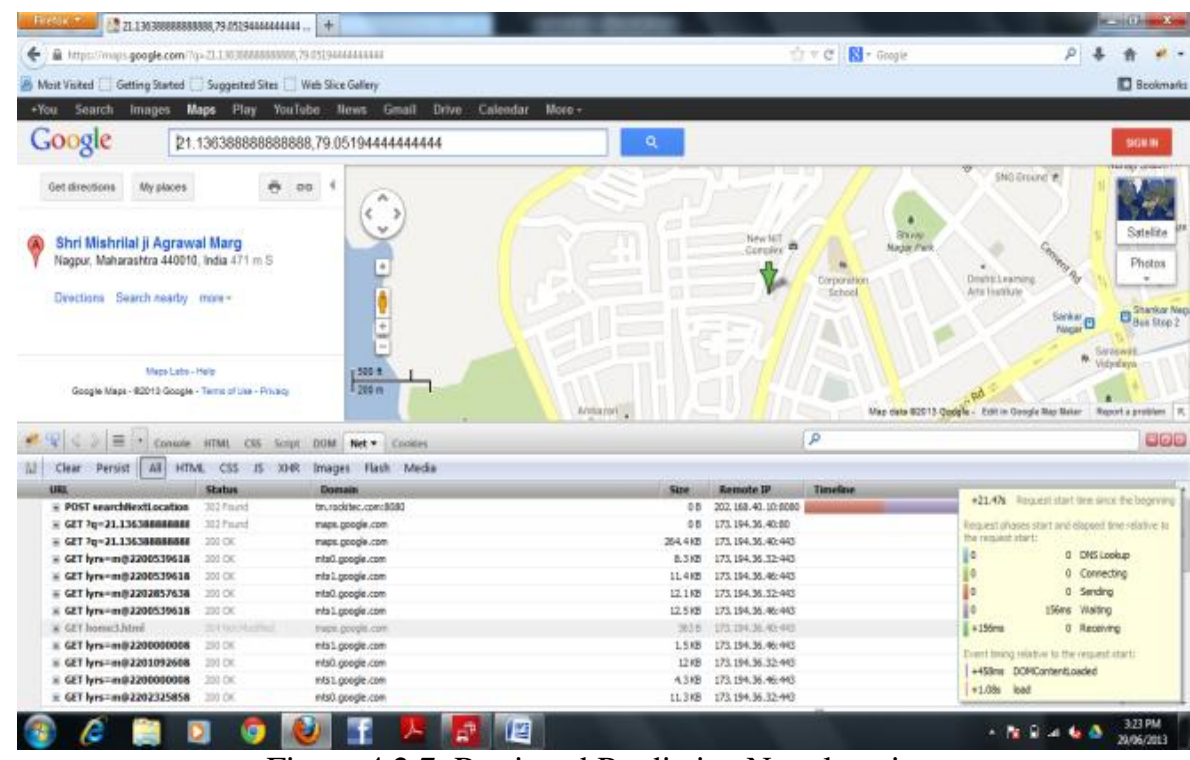

Figure 4.2.7: Retrieved Predictive Next location

\section{CONCLUSION}

CBIR approach via wavelet decomposition of images, followed by feature extraction and similarity match under F-norm theory. We compared the retrieval performance of D4 Wavelet, Haar wavelet schemes with the existing technique wavelet histograms. It turns out that D4 wavelet has greatly speeded retrieval as well as ensured enough recall rate comparable with its Haar wavelet and wavelet histogram. In addition, the progressive retrieval strategy helps to achieve flexible compromise among retrieval indices.

Finally we conclude from the results that wavelets achieve high retrieval performance in real time CBIR systems which will used in digital forensic [11]

\section{REFERENCES}

[1] Vijay Bagdi, Sulabha Patil, Dr. R.V.Dharskar,"Content Based Retrieval of Geoatagged Images using Wavelet as part of Digital Forensic",

[2] Vijay Bagdi, Sulabha Patil, Dr. R.V.Dharskar, "Analysis of Various CBIR techniques for Retrieving Forensically Viable Information from the Images", International Journal of Engineering Research \& Technology (IJERT), ISSN: 2278-0181, Vol. 2 Issue 1, January- 2013 
[3] Gulfishan Firdose Ahmed and Raju Barskar, "A Study on Different Image Retrieval Techniques in Image processing" International Journal of Computing and Engineering (IJSCE) ISSN:2232-2307, Volume-1, Issue-4, September 2011.

[4] Flickner, Metal (1995) "Query by image and video content: the QBIC system" IEEE Computer 28(9), 2332

[5] R. Brunelli and O. Mich, "Histograms Analysis for Image Retrieval," Pattern Recognition, Vol.34, No.8, pp1625-1637,2001.

[6] M. Adoram and M. S. Lew, "IRUS: Image Retrieval Using Shape," Proceedings of IEEE International Conference on multimedia Computing and System, Vol. 2, pp. 597-602,1999.

[7] K. Fukunaga, Introduction to Statistical Pattern Recognition, San Diego, CA, Academic Press, 1990.

[8] B. Brandshaw. "Semantic based image retrieval: probabilistic approach," proc, ACM Multimedia, ctober2000. http://www.cs.virginia.edu/papers/MIS03.pdf

[9] Flickner M, Sawhney H, N alblack W, et al. Query by image and video content: the QBIC system. IEEE Computer, 1995, 28 (9): 23 -32.

[10] Ripples in Mathematics: the Discrete Wavelet Transform by Arne Jense and Anders la Cour-Harbo, Springer, 2001

[11] Rizwan Ahmed, Dr. R. V. Dharaskar, Dr. V. M.Thakare, "Mobile Forensics: the study of collecting digital evidence from mobile devices", International Conference on Computer Networks and Security (ICCNS 2008), VIT, Pune, Sept 27-28, pp 246-253

[12] Mrs. Y.M.Latha, Dr. B.C.Jinga and V.S.K.Reddy "Content based Color image Retrieval via Wavelet Tranform” IJCSNS International Journal of Computer Science and Network Security, Vol, 7 No. 12, Dec'07

[13] Rui Y. \& Huang T. S., Chang S. F. "Image retrieval: current techniques, promising directions, and open issues". Journal of Visual Communication and Image Representation, 10, 39-62, 1999. 